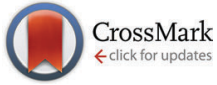

Cite this: J. Mater. Chem. B, 2016, 4, 6797

Received 2nd October 2016, Accepted 10th October 2016

DOI: 10.1039/c6tb02574a

www.rsc.org/MaterialsB

\section{Developing Mn-doped lead sulfide quantum dots for MRI labels†}

\author{
Lyudmila Turyanska, ${ }^{a b}$ Fabrizio Moro, ${ }^{a}$ Amalia Patanè, ${ }^{a}$ James Barr, ${ }^{a}$ \\ Walter Köckenberger, ${ }^{a}$ Alexander Taylor, ${ }^{c}$ Henryk M. Faas, ${ }^{c}$ Maxine Fowler, ${ }^{c}$ \\ Peter Wigmore, ${ }^{c}$ Rebecca C. Trueman, ${ }^{c}$ Huw E. L. Williams ${ }^{d}$ and Neil R. Thomas ${ }^{d}$
}

\begin{abstract}
Magnetic interactions of $\mathrm{Mn}^{2+}$ ions in lead sulfide ( $\mathrm{PbS}$ ) nanocrystals with protons in water are probed by NMR and MRI. A thin layer of capping molecules enables free solvent diffusion to the nanocrystal surface resulting in a decrease of proton relaxation times. Magnetic resonance imaging of neuronal cell pellets exposed to ( $\mathrm{PbMn}) \mathrm{S}$ at non-toxic concentrations demonstrates their prospects as MRI-labels.
\end{abstract}

Doping of colloidal semiconductor nanocrystals (Quantum Dots, QDs) with impurities has the potential to advance the use of QDs in a range of applications from optoelectronic devices to biomedical imaging. ${ }^{1,2}$ The optical stability and tuneability of the QD emission offer significant advantages for bioimaging and self-reporting drug delivery systems, particularly in the near-infrared (NIR) wavelength range 1000-1300 nm, where absorption of biological tissues and light scattering are favourable for signal penetration through deep tissues. ${ }^{2,3}$ On the other hand, doping with magnetic impurities ${ }^{4}$ in combination with the tuneable QD absorption could enable development of multifunctional labels for combined Magnetic Resonance Imaging (MRI) and fluorescence imaging. ${ }^{5}$ The complementarity of these imaging techniques, i.e. high spatial and temporal resolution of optical imaging and the non-invasive anatomical three dimensional (3D) imaging capability of MRI, could enable significant progress in medical diagnostics, for example, during surgical procedures, which require real-time image guidance and high resolution imaging. ${ }^{6}$

The advantages of colloidal semiconductor quantum dots for fluorescence imaging have been widely explored ${ }^{7,8}$ and particular interest has been recently focused on QDs with emission in the near-infrared wavelength range. ${ }^{9}$ On the other hand, the development of nanoparticles as MRI contrast agents has

\footnotetext{
${ }^{a}$ School of Physics and Astronomy, The University of Nottingham, NG72RD, UK.

E-mail: Lyudmila.Turyanska@nottingham.ac.uk

${ }^{b}$ School of Chemistry, University of Lincoln, LN6 7TS, UK

${ }^{c}$ Faculty of Medicine and Health Sciences, The University of Nottingham, NG72RD, UK

${ }^{d}$ Centre for Biomolecular Sciences, School of Chemistry, The University of Nottingham, $N G 72 R D, U K$

† Electronic supplementary information (ESI) available. See DOI: 10.1039/ c6tb02574a
}

mainly directed to iron oxide particles, ${ }^{5,10}$ Mn-clusters $^{11,12}$ and bi-metallic nanoparticles. ${ }^{13,14}$ However, these do not fluoresce. To achieve multifunctionality, different hybrid systems have been explored, including composite particles of QDs and iron oxides, ${ }^{5}$ and QDs with conjugated Gd(III)-chelates. ${ }^{15,16}$ These approaches significantly increase the size of the probes and thus the use of these nanostructures in medical imaging may be limited by inefficient transport through tissues and their clearance. ${ }^{17,18}$ Doping of colloidal QDs with transition metals ( $\mathrm{Mn}, \mathrm{Fe}$, etc.) could offer an alternative strategy with minimal changes in size or morphology, as demonstrated for visible $\mathrm{II}-\mathrm{VI}^{19,20}$ and I-III-VI ${ }^{21,22}$ QDs. In particular, Mn has been recently identified as a promising alternative to Gd in MRI. ${ }^{23}$ Mn-Based nanoparticles can have a relaxivity comparable to that of $\mathrm{Gd}(\mathrm{III})$ currently used in MRI as a contrast agent, ${ }^{24}$ but significantly lower intrinsic cytotoxicity. ${ }^{20}$ Although chelated Mn-complexes can also be employed in MRI, a Mn-doped QD would be less susceptible to chemical reactions and could be designed to combine within one structure magnetic properties with stable and tuneable absorption and emission, thus providing a promising route to multi-modal imaging. Furthermore, the nanoscale design of magnetic interactions within a QD has the potential to advance the use of nanoparticles in MRI and to open up new research directions in medical imaging.

Here we examine magnetic interactions in Mn-doped lead sulphide (PbS) semiconducting QDs capped with thioglycerols (TGL) and demonstrate that these NIR fluorescent QDs can be used as MRI labels (Fig. 1a and b). The capping molecules provide the nanocrystal with a thin shell, which enables the dispersion and solubilisation of the QDs in physiological solvents and allows free diffusion of the water molecules in close proximity to the QD surface, thus resulting in Mn-induced change of the magnetic resonance relaxation of hydrogen nuclei in surrounding water. We show that a Mn-doping of $\sim 5 \%$ results in a transverse relaxivity, $r_{2}=16 \mathrm{mM}^{-1} \mathrm{~s}^{-1}$, comparable to that of super-paramagnetic iron oxide particles. ${ }^{27}$ However, we note that the use of iron oxide particles for cell transplantation tracking and intra-operative guidance is limited 

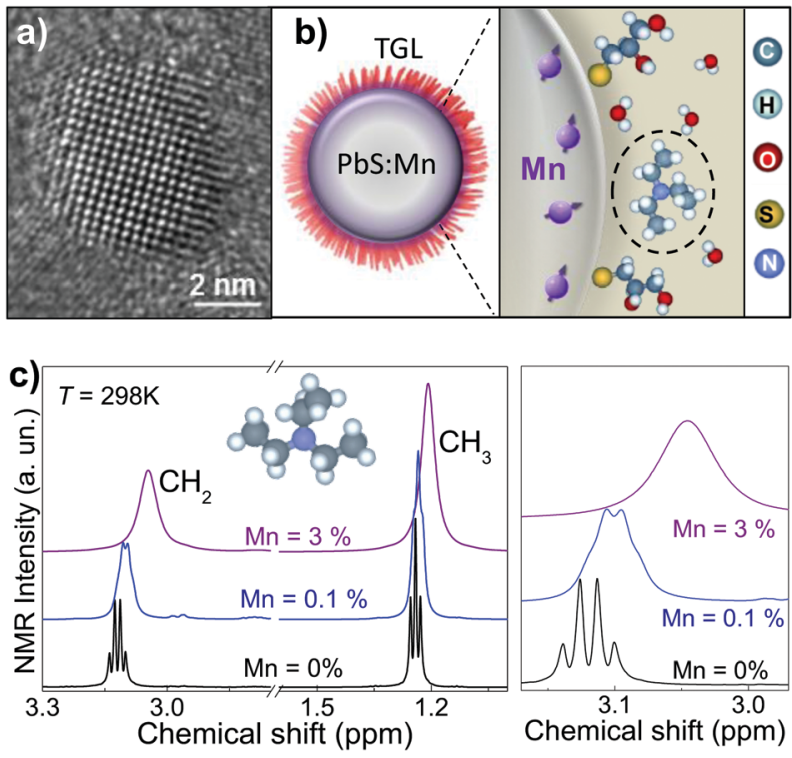

Fig. 1 (a) A typical high resolution TEM image of a Mn-doped PbS nanocrystal and (b) a schematic representation of a Mn-doped quantum dot capped with a layer of capping molecules (thioglycerol) in aqueous solution with trimethylamine (dashed circle) used to control the $\mathrm{pH}$. (c) NMR spectra of PbS and (PbMn)S QDs revealing resonance lines characteristic for triethylamine (inset).

due to the hypointensity generated in MRI images being indistinguishable from small bleeds. ${ }^{26}$ Thus Mn-doped nanoparticles could be advantageous. Here we demonstrate the effect of our nanocrystals on neuronal cell viability and show that at non-toxic concentrations $\left(<0.05 \mathrm{mg} \mathrm{mL}^{-1}\right)$ the QDs, uptaken by neuronal cells, alter significantly the water relaxation properties thus providing contrast in MRI. Since these nanoparticles are also fluorescent with emission tuneable in the NIR, ${ }^{28,29}$ they have potential for applications in dual in vivo imaging.

Colloidal Mn-doped PbS nanocrystals are synthesised in aqueous solution with Mn-content up to $7 \%$. A mixture of 1-thioglycerol and 2,3-dimercapto-1-propanol is used to stabilise the nanocrystals and to make them water soluble. Triethylamine $\left(\mathrm{Et}_{3} \mathrm{~N}\right)$ is used to control the $\mathrm{pH}$ of the QD solution. $\mathrm{Mn}^{2+}$ is incorporated as substitutional impurity into the $\mathrm{Pb}$-sublattice thus forming alloyed (PbMn)S nanocrystals with room temperature photoluminescence (PL) emission tuneable by Mn-content in the range 850-1200 $\mathrm{nm} .{ }^{25}$ Transmission electron microscopy (TEM) and high-resolution (HR) TEM images indicate that the QDs have average diameter $d=4.5 \pm 1.2 \mathrm{~nm}$, independent of Mn content, and confirm that alloyed nanocrystals retain high crystallinity (Fig. 1 and ESI, $\dagger$ Fig. S1).

NMR provides a powerful technique for determining the effect of paramagnetic impurities on proton spin relaxation time constants (i.e. $T_{1}$ and $T_{2}$ ) as well as a method for structural characterization. Here we use NMR spectroscopy to probe the interaction between the paramagnetic $\mathrm{Mn}^{2+}$ ions encapsulated into the QDs and the protons in surrounding molecules. In particular, we focus on the relaxation properties of protons of the $-\mathrm{CH}_{3}$ and $-\mathrm{CH}_{2}$ groups of $\mathrm{Et}_{3} \mathrm{~N}$ molecules present in the solvent. The NMR spectra of PbS and (PbMn)S QDs recorded at $T=298 \mathrm{~K}$ (Fig. 1c) show two intense and resolved peaks. By comparing these spectra to that of $\mathrm{Et}_{3} \mathrm{~N}$ in water (not shown), we assign the quartet at $3.12 \mathrm{ppm}$ and the triplet at $1.24 \mathrm{ppm}$ to the $-\mathrm{CH}_{2}$ and $-\mathrm{CH}_{3}$ groups, respectively. ${ }^{30}$ We attribute the multiplet splitting to the spin-spin scalar coupling $J=7.7 \mathrm{~Hz}$ for both $-\mathrm{CH}_{3}$ and $-\mathrm{CH}_{2}$, which is unchanged with respect to those of the pure $\mathrm{Et}_{3} \mathrm{~N}$ sample. However, for PbS QD solutions we observe a significant down-field chemical shift of the resonance peaks of $\mathrm{Et}_{3} \mathrm{~N}$ by $0.71 \mathrm{ppm}$ and $0.35 \mathrm{ppm}$ for $-\mathrm{CH}_{3}$ and $-\mathrm{CH}_{2}$ groups, respectively. We note that the chemical shift and $J$-couplings are independent of the proton concentration (i.e. $\mathrm{H}_{2} \mathrm{O} / \mathrm{D}_{2} \mathrm{O}$ ratio from $1: 0.1$ to $1: 120$ ) and $\mathrm{pH}$ of the solution. Incorporation of $\mathrm{Mn}$ in the QDs leads also to an up-field shift of $0.06 \mathrm{ppm}$ for $\mathrm{CH}_{2}$ and of $0.035 \mathrm{ppm}$ for $\mathrm{CH}_{3}$ groups, and broadening of the resonance lines. For a Mn-content larger than $0.1 \%$, the multiplet structures cannot be resolved. The broadening suggests that the local magnetic moment of the $(\mathrm{PbMn}) \mathrm{S}$ QDs induces paramagnetic relaxation on the protons of the $\mathrm{Et}_{3} \mathrm{~N}$ molecules.

Analysis of the proton relaxation times in $-\mathrm{CH}_{2}$ and $-\mathrm{CH}_{3}$ groups of $\mathrm{Et}_{3} \mathrm{~N}$ show a two orders of magnitude decrease from $T_{1}=2.40 \pm 0.09 \mathrm{~s}$ for $-\mathrm{CH}_{2}$ and $T_{1}=2.66 \pm 0.04 \mathrm{~s}$ for $-\mathrm{CH}_{3}$ groups in the PbS QD solution to $0.024 \pm 0.003 \mathrm{~s}$ and $0.027 \pm$ $0.002 \mathrm{~s}$, respectively, for $(\mathrm{PbMn}) \mathrm{S}$ with $\mathrm{Mn}=3 \%$. The length of the capping molecules used to terminate the growth of the nanocrystals is $<0.5 \mathrm{~nm}$ and allows free diffusion of $\mathrm{Et}_{3} \mathrm{~N}$ molecules to the QD surface resulting in a significant decrease of the relaxation times via paramagnetic relaxation effects. Similar effects are observed for the relaxation properties of water proton spins in MRI. As shown in Fig. 2a, our MRI studies show that $T_{1}$ and $T_{2}$ relaxation times are significantly decreased by an increasing Mn-content. For solutions with $7 \% \mathrm{Mn}$ and QD concentration of $5 \mathrm{mg} \mathrm{mL}^{-1}$, we observe a decrease of $T_{1}$ and $T_{2}$ by one and two orders of magnitude, respectively, compared to identical control samples with $0 \% \mathrm{Mn}$. This increase of relaxation rates by $\sim 100$-times is comparable to the change estimated in our NMR studies. We note that in undoped PbS QD solutions the relaxation rate of the water protons is not noticeably affected by the presence of the nanocrystals. As shown in the inset of Fig. 2a, the Mn-induced change of $T_{1}$ and $T_{2}$ provides significant contrast in MRI images of QD solutions.

We envisage that Mn-ions in closer proximity to the QD surface affect predominantly the relaxation of the surrounding water protons. By assuming a uniform distribution of $\mathrm{Mn}$ throughout a spherical nanocrystal, we estimate that the number of Mn-ions within a distance of $0.5 \mathrm{~nm}$ from the QD surface increases from $\sim 25$ for $\mathrm{Mn}=1 \%$ to $\sim 150$ for $\mathrm{Mn}=7 \%$. On the other hand, the broadening of the NMR lines and the non-linear decrease of $T_{1}$ and $T_{2}$ with increasing Mn-content indicate much stronger effects at low $\mathrm{Mn}$ content $(\mathrm{Mn}<1 \%)$, thus suggesting the preferential location of Mn close to the periphery of the nanocrystal. ${ }^{31}$ Self-purification mechanisms ${ }^{4}$ can lead to impurity out-diffusion resulting in greater impurity content in the proximity of the QD surface compared to the centre. ${ }^{32}$ The greater Mn-dependent reduction of $T_{1}$ and $T_{2}$ times at low Mn compared to higher Mn content indicates a saturation of the concentration of Mn-ions close to the QD surface. 

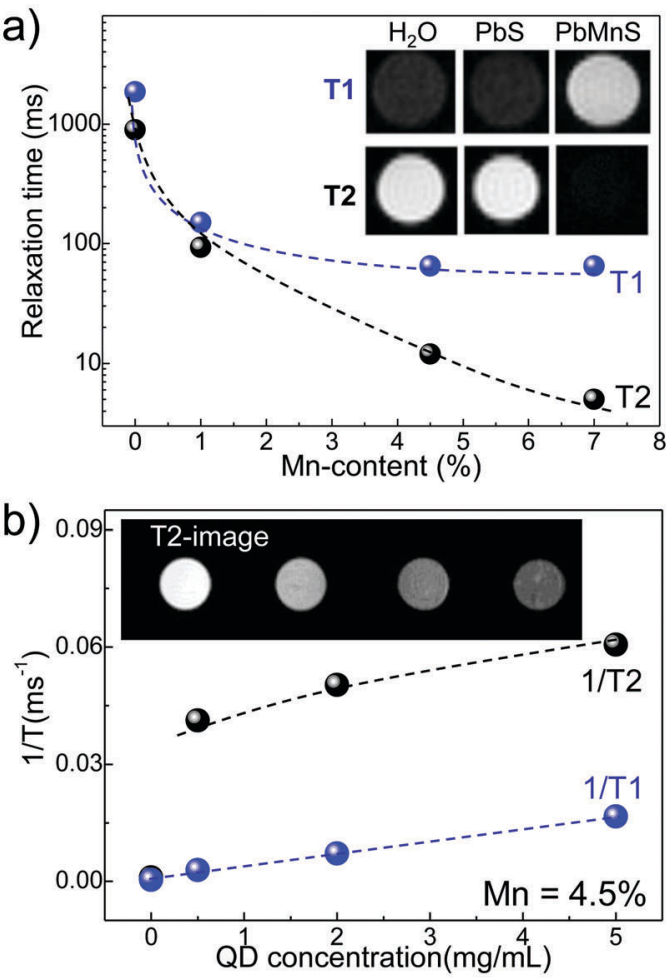

c)

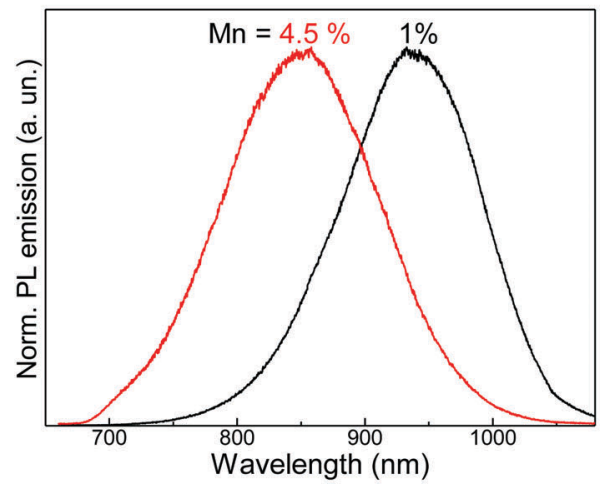

Fig. 2 (a) Effect of Mn-content in the PbS nanocrystals on the MRI relaxation times. Inset: $T_{1}$ and $T_{2}$ images for water and aqueous solutions of $\mathrm{PbS}$ and $(\mathrm{PbMn}) \mathrm{S}(\mathrm{Mn}=4.5 \%)$ QDs. (b) Effect of representative QD concentration on water relaxation times for $\mathrm{QDs}$ with $\mathrm{Mn}=4.5 \%$. Inset: Corresponding $T_{2}$-weighted images of QD solutions. (c) Room temperature normalized photoluminescence (PL) spectra of QDs with Mn content of $1 \%$ and $4.5 \%$.

Representative $T_{1}$ and $T_{2}$ times of serial dilution of (PbMn)S QDs with $4.5 \%$ and corresponding $T_{2}$-weighted images are shown in Fig. 2b. To facilitate the comparison with other works, we assess the relaxivity per Mn concentration: at room temperature and magnetic field $B=7 \mathrm{~T}$, we find $r_{1}=5 \mathrm{mM}^{-1} \mathrm{~s}^{-1}$ and $r_{2}=16 \mathrm{mM}^{-1} \mathrm{~s}^{-1}$, for $T_{1}$ and $T_{2}$, respectively. The paramagnetism of the QDs, water accessibility and rotational dynamics are likely causes for the more pronounced $T_{2}$ effect compared to $T_{1}$ in these QDs. To assess the effect of environment viscosity, and thus water diffusion, on the QD-induced changes of relaxivity, we performed the MRI studies of QDs dispersed in $0.5 \%$ agarose and found no noticeable differences in the values of $T_{1}$ and $T_{2}$ compared to those in water. This suggests that similar effects should be observed in cells and/or tissues. Our investigations demonstrate relaxivity values that are similar or greater than those reported for Mn-doped II-VI QDs. ${ }^{19,20}$ Remarkably, the relaxivity values of $r_{2}$ are similar to those of Fe-based superparamagnetic nanoparticles (SPIONs) ${ }^{26}$ and only 10-times lower compared to commercial Fe-based contrast agents. ${ }^{33}$ Since our Mn-doped QDs are significantly smaller $(d \sim 5 \mathrm{~nm})$ compared to clinically approved Fe-based particles $(d>100 \mathrm{~nm}),{ }^{33}$ the greater cellular uptake combined with the large relaxation rates could provide better contrast in MRI. Also, following the incorporation of $\mathrm{Mn}$, the QDs remain optically active in the NIR wavelength range, as shown in Fig. 2c for $\mathrm{Mn}=1$ and $4.5 \%$.

Imaging of neural cells is of great importance for assessing cell transplant therapies ${ }^{34}$ and Mn-based contrast agents have been considered of great promise. ${ }^{11,35,36}$ One of the main concerns in applications of the QDs is their penetration through the blood-brain barrier, which could be controlled by functionalization of the nanocrystals with cell penetration peptides. $^{37}$ To investigate the potential of our (PbMn)S QDs for neuronal imaging, we have exposed the N2a mouse neuroblastoma cells to QDs with Mn content of $4.5 \%$ for 24 hours. Our TEM images reveal localized QD clusters in the cytoplasm indicating that the cellular uptake occurs via endocytosis. ${ }^{38,39}$ The cellular uptake is sufficient to allow photoluminescence/ absorption of the QDs to be detected in cells and cell pellets. For MRI, the cell pellets of equal volume in Eppendorf tubes were positioned at the centre of a $7 \mathrm{~T}$ magnet. $T_{2}$-Weighted MRI images of cell pellets (Fig. 3) reveal a QD-induced contrast for QD treatment concentrations of $0.005 \mathrm{mg} \mathrm{mL}^{-1}$ and $0.01 \mathrm{mg} \mathrm{mL}^{-1}$. The 3-fold change in MRI intensity for the cells exposed to (PbMn)S QDs compared to the control sample is easily discernible by eye and comparable to the contrast achieved with SPIONs ${ }^{23}$ and in Mn-enhanced MRI. ${ }^{11,36}$

We note that the QD concentrations used in MRI are nontoxic to the cell. We compare the cytotoxicity of (PbMn)S in two representative cell lines: N2a and WGE. The N2a is an immortal neural cell line derived from mouse neuroblastoma tumours

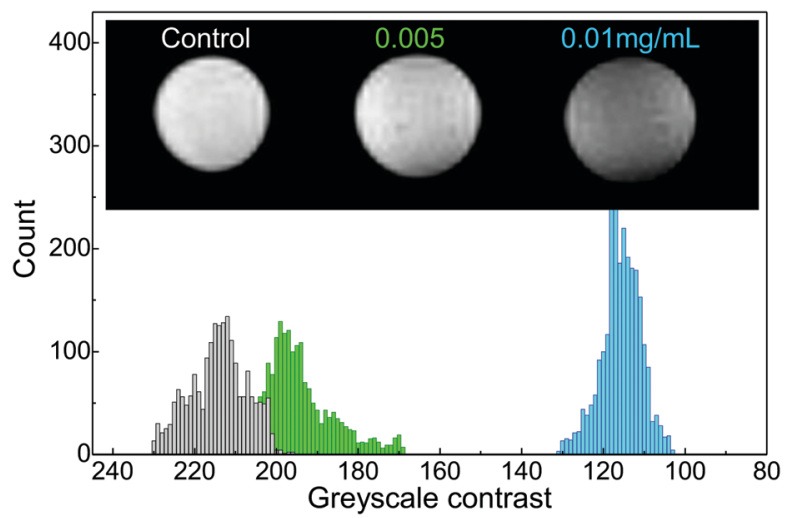

Fig. $3 T_{2}$-Weighted image of neuronal cell pellets following $24 \mathrm{~h}$ exposure to $(\mathrm{PbMn}) \mathrm{S}$ QDs $(\mathrm{Mn}=5 \%)$ and a corresponding histogram of the detected MRI signal. 

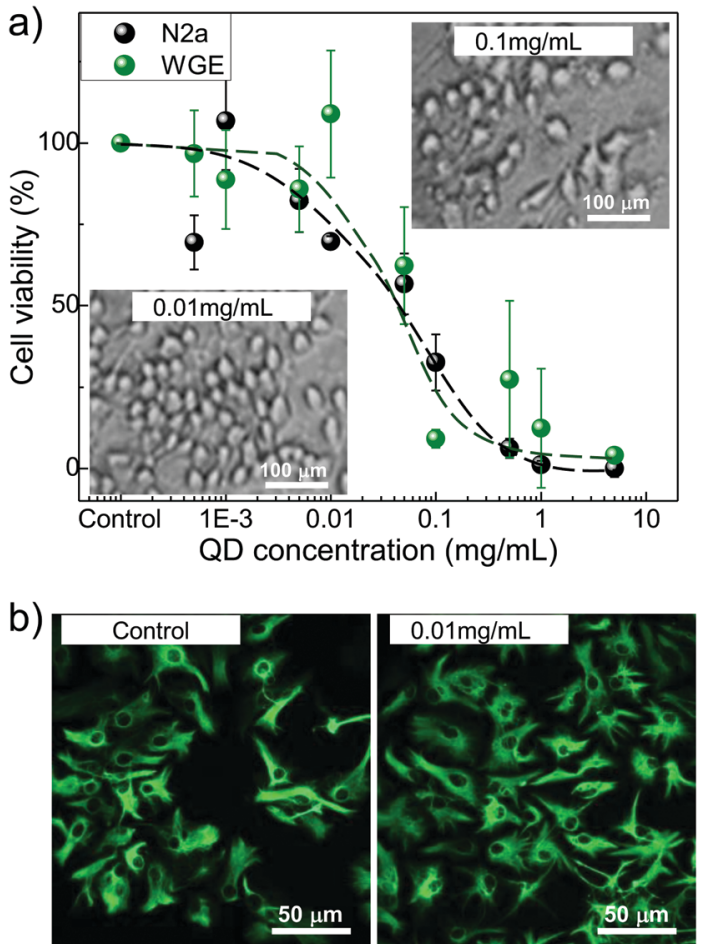

Fig. 4 (a) MTT analysis of cell viability after 48 hours of QD treatment $(\mathrm{Mn}=1 \%)$. All experiments were repeated 3 -times with 6 replicates each; error bars represent standard error of the mean. Inset: Optical images of N2a cells following $48 \mathrm{~h}$ exposure to different QD concentrations. (b) GFAP immunocytochemistry of primary cells (WGE) untreated and following 7 days exposure to QDs.

that can be differentiated into neurones. The primary WGE cells were dissected from developing mouse embryos at E14 and have the potential to become neurones or glial cells of the adult striatum. For MTT analysis to test neuronal viability in vitro, the $\mathrm{N} 2 \mathrm{a}$ and WGE cells were treated with (PbMn)S QDs for up to $72 \mathrm{~h}$ (Fig. 4a).

Additionally, primary cells were assessed over 7 days following exposure to QDs. The highest dose which did not induce significant cell death at any time point 24,48 and 72 hours and 7 days) in the primary cell cultures was determined to be $0.01 \mathrm{mg} \mathrm{mL}^{-1}$, and there was a trend towards N2a cells being less resilient to the effects of the QDs. The growth inhibition values for $\mathrm{N} 2 \mathrm{a}$ and WGE cells $\left(\mathrm{GI}_{50}=0.05 \mathrm{mg} \mathrm{mL}{ }^{-1}\right)$ are lower compared to fibroblast cells, ${ }^{35}$ indicating higher sensitivity of neuronal cells to QD induced toxicity. Optical microscopy assessment of N2a cells revealed reduced cell density and loss of membrane integrity at QD treatment concentrations above $0.1 \mathrm{mg} \mathrm{mL} \mathrm{m}^{-1}$, indicative of cell death (inset in Fig. 4a). Following 7 day treatment with $0.01 \mathrm{mg} \mathrm{mL}^{-1}$ (PbMn)S QDs (Fig. 4b) GFAP immunocytochemistry analysis revealed no alterations to the differentiation and morphology of cells. However, at treatment concentration of $>0.05 \mathrm{mg} \mathrm{mL}^{-1}$, cell death occurred and surviving GFAP neurones exhibited altered morphology. There is no noticeable release of Mn-ions from the QD since the QD induced toxicity levels are comparable to those of PbS QDS and are independent of the Mn-content. Recent studies raised concerns about possible ROS-induced cell damage (e.g. DNA damage) following exposure to nanoparticles. ${ }^{40,41}$ We note that at concentrations below $\mathrm{GI}_{50}$ values we do not observe any QD-induced changes to cell viability and proliferation as probed by MTT assay, cell cycle and annexin V studies, and ROS. ${ }^{28,38}$ Our previous acute toxicity studies have demonstrated that PbS-based QDs do not accumulate in the organs, are well tolerated by mice, and their NIR photoluminescence can be imaged in vivo. ${ }^{34}$

\section{Conclusions}

In conclusion, we have demonstrated that Mn-doped PbS QDS can be used as biocompatible MRI probes. By encapsulating the nanocrystals with a thin layer of capping molecules, we enable the free diffusion of water molecules to the QD surface resulting in a decrease of proton relaxation times and contrast in MRI. At the concentrations where these QDs do not exhibit cellular toxicity, the $T_{2}$-weighted images reveal a 3 -fold change of MRI intensity. The combination of paramagnetism and fluorescence emission in the near-infrared wavelength range of low absorption of biological tissues makes these QDs promising candidates for dual medical imaging. Presently, specific targeting of these QDs is being explored and combined in vivo mice imaging is being developed in conjunction with extension of the detection range of current fluorescence live animal imaging systems to the NIR.

\section{Experimental section}

\section{Morphological, optical and NMR studies}

For the transmission electron microscopy (TEM) study, the nanocrystals were deposited on a graphene-oxide coated grid and TEM images were recorded on a JEOL1200EX microscope operating at $120 \mathrm{kV}$.

Optical properties of quantum dots were measured in solution and in agarose gel pellets. The optical excitation was provided by the $532 \mathrm{~nm}$ line of a solid state laser and an excitation power in the range $P=10-100 \mathrm{~W} \mathrm{~m}^{-2}$. The luminescence was dispersed by a $150 \mathrm{~g} \mathrm{~mm}^{-1}$ grating and detected by either a nitrogen-cooled (InGa)As array photodiode or a chargecoupled device (CCD). We note that aqueous solutions of PbS:Mn are stored at $T=4{ }^{\circ} \mathrm{C}$ under nitrogen atmosphere and are stable over a period of at least 3 month. The colloidal stability of aqueous nanoparticles can be altered by oxidation of the surface and/or removal of the capping ligands and care should be taken when subjecting QDs to high temperatures $\left(T>50{ }^{\circ} \mathrm{C}\right)$ and/or low pH environment. The Nuclear Magnetic Resonance (NMR) data were collected at $600 \mathrm{MHz}$ on a Bruker Avance III spectrometer at $298 \mathrm{~K}$ on QDs dispersed in $\mathrm{H}_{2} \mathrm{O}: \mathrm{D}_{2} \mathrm{O}$ (1:9). Solvent suppression was achieved using excitation sculpting, where required. $T_{1}$ experiments were conducted with an inversion recovery sequence: $\pi$-delay $(T)-\pi / 2$-free induction decay. Data were acquired as a pseudo $2 \mathrm{D}$ and the relaxation delay was set at $>5 \times T_{1}$ to facilitate complete recovery between 
transients. Data were phased and baseline corrected prior to integration using TOPSPIN 3 software.

\section{Magnetic resonance imaging (MRI)}

All MRI experiments were performed on a horizontal Bruker Biospec $7 \mathrm{~T}$ Avance III scanner (Bruker Biospin MRI GmbH, Ettlingen, Germany) with a $7 \mathrm{~cm}$ transmit/receive volume coil. QD solutions were extensively dialysed to remove the residual free Mn-ions. $1 \mathrm{~mL}$ of samples in Eppendorf tubes were positioned in a polystyrene holder. Relaxometry measurements were performed by taking a gradient echo localiser scan to establish central positioning of the samples in the magnet, followed by a Rapid Acquisition with Relaxation Enhancement Variable Time of Repetition (RARE-VTR) fast spin echo $T_{1} / T_{2}$ scan. Scanner software (Paravision 5.1) was used to determine a $0.15 \mathrm{~cm}^{2}$ region of interest for each sample and to obtain relaxation values. A series of eight echo times (TE from $6.5 \mathrm{~ms}$ to $240 \mathrm{~ms}$ ) and inversion times (TI from $30 \mathrm{~ms}$ to $13 \mathrm{~s}$ ) were optimised, and $T_{1}$ and $T_{2}$ relaxation curves were plotted. The following parameters were used for all relaxation measurements: matrix size $=$ $128 \times 128$, field of view (FOV) $80 \mathrm{~mm} / 70 \mathrm{~mm} \times 50 \mathrm{~mm}$, slice thickness $1.5 \mathrm{~mm}$ and acquisition time $\tau_{\mathrm{aq}} \sim 11 \mathrm{~min}$.

\section{MTT and immunofluorescence assays}

The cells were seeded into 96-well plates at a density of $6 \times 10^{3}$ per well and allowed 24 hours to adhere before treatment with serially diluted QD solutions (from $5 \mathrm{mg} \mathrm{mL}^{-1}$ to $0.5 \mu \mathrm{g} \mathrm{mL}^{-1}$ ) for up to $72 \mathrm{~h}$. Control wells received the vehicle alone. Following exposure, MTT was added to each well at a final concentration of $5 \mathrm{mg} \mathrm{mL}{ }^{-1}$ and incubated at $37^{\circ} \mathrm{C}$ for $30 \mathrm{~min}$. Supernatants were aspirated and cellular formazan solubilised by the addition of DMSO and absorbance was read at $550 \mathrm{~nm}$. Primary striatal cells from mouse embryos (WGE) were seeded at a density $5 \times 10^{4}$ per well and were exposed to QDs $(0,0.01$, 0.05 and $0.1 \mathrm{mg} \mathrm{mL}^{-1}$ ) for 7 days. Then the cells were fixed with 4\% paraformaldehyde for 15 minutes, washed several times in PBS, permeabilised for 10 minutes (PBS with $0.25 \%$ Triton $\mathrm{X}-100)$ and blocked with $10 \%$ goat serum, $0.25 \%$ Triton X-100 and 0.3 $\mathrm{M}$ glycine in PBS for 1 hour. Primary antibodies were diluted as follows: NeuN (to visualise neuronal cell nuclei, monoclonal; $1: 2000$ ) and GFAP (to visualise glial cells, polyclonal; $1: 1000$ ) in $1 \%$ BSA, 0.3 M glycine, $0.25 \%$ Triton $\mathrm{X}-100$ in PBS and were incubated overnight at $4{ }^{\circ} \mathrm{C}$. The cells were washed extensively with PBS and then incubated in the dark with the secondary antibody $(1: 500)$ in $1 \%$ BSA, $0.25 \%$ Triton $\mathrm{X}-100$ and $0.3 \mathrm{M}$ glycine in PBS for 1 hour. After further three PBS washes, the cells were counter stained with DAPI $(1: 1000)$, washed in distilled water. The cover slip was applied using DABCO mounting medium before being viewed under a Nikon microscope.

\section{Acknowledgements}

The work was supported by funding from The Leverhulme Trust (Grant No. RPG-2013-242), NC3Rs/EPSRC (Grant No. NC/L001861/1), the EPSRC Impact Acceleration Account (EP/K503800/1) and The University of Nottingham. We acknowledge useful discussions and assistance with TEM and confocal imaging from Dr D. McLean and Dr M. W. Fay.

\section{Notes and references}

1 R. Saran and R. J. Curry, Nat. Photonics, 2016, 10, 81-92.

2 G. Hong, S. Diao, J. Chang, A. L. Antaris, C. Chen, B. Zhang, S. Zhao, D. N. Atochin, P. L. Huang, K. I. Andreasson, C. J. Kuo and H. Dai, Nat. Photonics, 2014, 8, 723.

3 R. Koole, W. J. M. Mulder, M. M. van Schooneveld, G. J. Strijkers, A. Meijerink and K. Nicolay, Wiley Interdiscip. Rev.: Nanomed. Nanobiotechnol., 2009, 1, 475.

4 D. J. Norris, A. L. Efros and S. C. Erwin, Science, 2008, 28, 1776.

5 L. Jing, K. Ding, S. V. Kershaw, I. M. Kempson, A. L. Rogach and M. Gao, Adv. Mater., 2014, 26, 6367.

6 J. Jin, Z. Xu, Y. Zhang, Y. J. Gu, M. H. Lam and W. T. Wong, Adv. Healthcare Mater., 2013, 2(11), 1501.

7 M. X. Zhao and E. Z. Zeng, Nanoscale Res. Lett., 2015, 10, 171.

8 X. Michalet, F. F. Pinaud, L. A. Bentolila, J. M. Tsay, S. Doose, J. J. Li, G. Sundaresan, A. M. Wu, S. S. Gambhir and S. Weiss, Science, 2005, 307, 538.

9 T. Jin and Y. Imamura, ECS J. Solid State Sci. Technol., 2016, 5, R3138-R3145.

10 L. Zhang, Y. Wang, Y. Tang, Z. Jiao, C. Xie, H. Zhang, P. Gu, X. Wei, G.-Y. Yang, H. Gu and C. Zhang, Nanoscale, 2013, 5, 4506.

11 J. Moraes Malheiros, F. Fernandes Paiva, B. Monteiro Longo, C. Hamani and L. Covolan, Frontiers in Neurology, 2015, 6(161), 1-10.

12 M. Kueny-Stotz, A. Garofalo and D. Felder-Flesch, Eur. J. Inorg. Chem., 2012, 1987.

13 P. C. Naha, A. A. Zaki, E. Hecht, M. Chorny, P. Chhour, E. Blankemeyer, D. M. Yates, W. R. Witschey, H. Litt, A. Tsourkas and D. P. Cormode, J. Mater. Chem. B, 2014, 2, 8239.

14 P. C. Naha, K. C. Lau, J. C. Hsu, M. Hajfathalian, S. Mian, P. Chhour, L. Uppuluri, E. S. McDonald, A. D. A. Maidment and D. P. Cormode, Nanoscale, 2016, 8, 13740.

15 Q. An, J. Liu, M. Yu, J. Wan, D. Li, C. Wang, C. Chen and J. Guo, Small, 2015, 11, 5675-5686.

16 C.-L. Huang, C.-C. Huang, F.-D. Mai, C.-L. Yen, S.-H. Tzing, H.-T. Hsieh, Y.-C. Ling and J.-Y. Chang, J. Mater. Chem. B, 2015, 3, 651.

17 M. Longmire, P. L. Choyke and H. Kobayashi, Nanomedicine, 2008, 3(5), 703.

18 H. S. Choi, W. Liu, P. Misra, E. Tanaka, J. P. Zimmer, B. I. Ipe, M. G. Bawendi and J. V. Frangioni, Nat. Biotechnol., 2007, 25, 1165.

19 V. K. Sharma, S. Gokyar, Y. Kelestemur, T. Erdem, E. Unal and H. V. Demir, Small, 2014, 10(23), 4961.

20 S. Wang, B. R. Jarrett, S. M. Kauzlarich and A. Y. Louie, J. Am. Chem. Soc., 2007, 129, 3848. 
21 K. Ding, L. Jing, C. Liu, Y. Hou and M. Gao, Biomaterials, 2014, 35, 1608-1617.

22 G. Sitbon, S. Bouccara, M. Tasso, A. Francois, L. Bezdetnaya, F. Marchal, M. Beaumonte and T. Pons, Nanoscale, 2014, 6, 9264-9272.

23 E. M. Gale, I. P. Atanasova, F. Blasi, I. Ay and P. Caravan, J. Am. Chem. Soc., 2015, 137, 15548.

24 J. G. Penfield and R. F. Reilly Jr., Nat. Clin. Pract. Nephrol., 2007, 3, 654-668.

25 M. Corti, A. Lascialfari, E. Micotti, A. Castellano, M. Donativi, A. Quarta, P. D. Cozzoli, L. Manna, T. Pellegrino and C. Sangregorio, J. Magn. Magn. Mater., 2008, 320, e320.

26 E. Tarulli, J. D. Chaudhuri, V. Gretka, A. Hoyles, C. M. Morshead and G. J. Stanisz, J. Magn. Reson. Imaging, 2013, 37(6), 1409.

27 J. Xiao, X. M. Tian, C. Yang, P. Liu, N. Q. Luo, Y. Liang, H. B. Li, D. H. Chen, C. X. Wang, L. Li and G. W. Yang, Sci. Rep., 2013, 3, 3424.

28 L. Turyanska, F. Moro, A. N. Knott, M. W. Fay, T. D. Bradshaw and A. Patanè, Part. Part. Syst. Charact., 2013, 30, 945.

29 L. Turyanska, R. J. A. Hill, O. Makarovsky, F. Moro, A. N. Knott, O. J. Larkin, A. Patanè, A. Meaney, P. C. M. Christianen, M. W. Fay and R. J. Curry, Nanoscale, 2014, 6(15), 8919.
30 SDBSWeb, http://sdbs.db.aist.go.jp, National Institute of Advanced Industrial Science and Technology.

31 F. Moro, L. Turyanska, J. Wilman, H. E. L. Williams, A. J. Fielding and A. Patanè, Nano Lett., 2016, 16, 6343-6348. 32 F. Moro, L. Turyanska, J. Granwehr and A. Patanè, Phys. Rev. $B, 2014$, 90, 205428.

33 Y.-X. J. Wang, Quant. Imaging Med. Surg., 2001, 1(1), 35.

34 M. Modo, J. S. Beech, T. J. Meade, S. C. R. Williams and J. Price, NeuroImage, 2009, 47, T133.

35 A. C. Silva, J. H. Lee, I. Aoki and A. P. Koretsky, NMR Biomed., 2004, 17, 532.

36 R. Pautler, A. C. Silva and A. P. Koretsky, Magn. Reson. Med., 1998, 40, 740.

37 S. Santra, H. Yang, P. H. Holloway, J. T. Stanley and R. A. Mericle, J. Am. Chem. Soc., 2005, 127, 1656.

38 T. D. Bradshaw, M. Junor, A. Patanè, P. Clarke, N. R. Thomas, M. Li, S. Mann and L. Turyanska, J. Mater. Chem. B, 2013, 1, 6254.

39 L. Turyanska, T. D. Bradshaw, J. Sharpe, M. Li, S. Mann, N. R. Thomas and A. Patanè, Small, 2009, 5(15), 1738.

40 K. Bhattacharya, P. C. Naha, I. Naydenova, S. Mintova and H. J. Byrne, Toxicol. Lett., 2012, 215, 151.

41 P. C. Naha, M. Davoren, F. M. Lyng and H. I. Byrne, Toxicol. Appl. Pharmacol., 2010, 246, 91. 